\title{
LISTA DE LOS MIEMBROS DE LA SOCIEDAD GEOLOGICA MEXICANA HASTA 1937
}

Nombre.
Domicilio.

- Sr. Dr. Rafael Aguilar y Santillán4 Morelia 99. México. D. F.

* Sr. Dr. José G Aguilera, Presi dente de la Sociedad Geológica Mexicana.. .............. $7^{\text {a }}$ Gahino Barreda 139. Méxieo, D. F.

Sr. Ing. Juan Allera. ... .........erdo de Tejada 1. Tuxpan, Ver.

Sr. Ing Carlos Almazán........ Isábel la Católica 85. Altos, Méxieo, D. F.

* Sr. Manuel Alvarez Jr. ........Pedro Baranda 11. México, D. F.

Sr. Ing. A. R. V. Arellano. ..... Apartado 150, Tampico, Tamps. Sr. Ing. Ernesto Azcón. ... . . Cía. Minera de Peñoles, S. A. Apartado 686, México, D. F.

Sr. Tng. Alfonso Barnetehe. .... Puebla 315. México, D. F.

* Sr. Luis Blásquez. . .......... León de los Aldamas 43. México, D. F.

* Sr. Ing. Ignacio S. Bonillas...... Edificio Boker, $\Lambda$ v. 16 de Sep. 58. Desp. 202-206. Méxieo, D. F.

Sr. Ing. Mauricio M. Breen. ...Av. Madero 26. Méxieo, D. F.

* Sr. Ing. B. P. Burchard........ Apartado 257. Monterrey, N. L.

"Sr. R. H. Burrows............ Apartado 405. San José, Costa Rica.

Sr. Ing. Salvador Cárdenas. .... Gúa. Carboniffera de Palau, Coah.

Sr. Ing. Arturo Chippendale... Paseo de la Reforma 427. México, D. F.

- Sucios activos fudadores.

*-Socios protectores. 
Nombre.

Domicilit.

* Sr. Prof. Francisco Contreras...7" de Zaragoza 11. México. D. F.

* Sr. Rodolfo del Corral... . . . . . 3. Sta. María la Ribera 107. México, D. F.

Cía. Mex. de Petróleo "El Aguila"', S. A., (Operating See-

tion-Head Office)... .. ... Apartado 113-bis., México,

D. F.

Sr. Ing. Jorge L. Cumming.......Av. Amsterdam 182. México. I). F.

Sr. Ing. Federico Deschamps.... Revillagigedo 47, Depto. 7, México, D, F.

* Sr. Ing. Enrique Díaz Lozano. . Guadalajara 90. México, D. F. Sr. Ing. A. R. Fdelen..........American Smetting \& Refi. ning Co. Madero 55. Méxi«o, D. F.

* Sr. Bernardo Eguía Lis.. .... Priv. del Recreo 30. Atzcapotzaleo, D. F.

* Sr. Prof. Luis Espino Flores..... Calle del Nogal 51. México; D. F.

Sr. Ing. Julio Flebbe........ Depto, de Fxploración. Sría. do la Eeon. Nac. Filomeno Matil 8. México, D. F.

* Sr. Ing. Teodoro Flores................. Gerrada de la Paz 27. Taeubaya, D. F.

* Srita. Clara Flores Covarrubias...... Cerrada de la Paz 27. 'Tacubaya, D. F.

* Sr. Ing. Tuis Flores Covarrubias.Cerrada de la Paz 27, Tacabayá, D. F.

* Sr. Ing. Vicente Gálvez........ . Depto, de Fxploración. Sría. de la Eeon, Nac, Filomeno Mata 8. México, D. F.

Sr. Ing. Valentín Gama........Av. Chapultepec 27. México, D. F.

Sr. Ing. Jenaro P. Gareía....... Av. Juárez 40. México, D. F.

* Sr. Ing. Germán García Lozano. Calle de Tepie 33 C., México, D. F.

Sr. Ing. Manues F. Garrido..... Tuondres 9. Colonia " El Mirador", Monterrey, N. L .

Sr. Ing. Juan B. Gibson....... Calle del Bajío 233. Méxieo, D. F. 
Nombre.

Domicilio.

Sr. K. Goldschmid. . ........ Gía. Mex. de Petróleo "El A guila" Apartado 150. Tampico, Tamps.

Sr. Ing. Luis Gómez Chico..... Cía. Minera Real del Monte. Pachuea, Hgo.

Sr. Ing. Rámón Gómez Tagle... Subjefe deI Depto. de Exploración, Sría. de la Eeon. Nac. Filomeno Mata 8. México, D. F.

* Sr. Enrique M. González...... Tesorero de Ia Soc. Geol. Me. xicana $6^{\circ}$ Calle del Ciprós 176. Méxieo, D. F.

Mr. Thomas Franeis Grimsdale. C/o Lloyds Bank Ltd. 263. lottenham. Court Road. London, W. 1. England.

* Sr. Ing. Jenaro González R.... Calle Fugenia 709. Col. del Valle, Móxico, D. F.

Sr. Oscar Grosslicht.. .........v. de la Paz 705. Guadalajara, Jal,

Sr. Tng. Enrique Guerra y Aguilar.. ...............Mexiamora No 6. Guanajuato Gto.

Sr. Walter H. Hegwein........Apartado 113 bis., México, D. $\mathrm{F}$.

* Sr. Ing. Apolinar Hernández. . .4.4 calte Santa María la Ribe. ra 89. México, D. F.

Sr. Ing Luis Hijar y IIaro..... Tamaulipas 247-A. Tacubaya, D. $\mathbf{F}$.

Sr. Misakichi Hisazumi.. ..... Geological Survey, Gowernment General of ''aiwan. Japón.

Sr. Ing Luis G. Jiménez....... 13 Mérida 241, México, D. F. Sr. Dr. Lewis B. Kellum........M us a $\mathrm{m}$ of Paleontology. Ann Arbor, Mich. U. S. A.

* Sr. Hermión Larios. . ........ Calle del Pjno 74, México. D. F.

* Sr. Ing. Pedro Landero.........Calle de Niza 66. México, D. F.

Sr. Ing. Amadeo Larralde...... Calle de Tepie 33-A., México, D. $\mathrm{F}$.

* Sr. Fabiár León.. .. ........ Dr. Montes de Oca 6. Méxieo, D. F. 
Nombre.

Domicilio.

Sr. Ing. José I ópez Martínez... Calle de Monclova 38. México, D. F.

Sr. Ing Andrés López Vázquez.. Riehmond Petroleum Co., Balderas No. 823, México, D. F.

* Sr. Jesús Martínez Portillo...... Prosecretario de la Soeiedad Geol. Mex. $6^{\mathrm{a}}$ ealle del Ciprés 176. México, D. F.

* Sr. Dr. Federico K. G. Müillerried. Calle de Córdoba 197. Méxieo, D. F.

Sr. Dr. A. C. Noé............ The University of Chicago, Chicago, Ill. U. S. A.

Sr. Ing. Alfonso de la $0 . \ldots \ldots$. Calle de Sor Juana Inés de 'la Cruz, A. México, D. F.

* Sr. Ing. Ezequiel Ordóñez, Secretario de la Sociedad Geolónica Mexicana.. .. .. .......... Abraham González 79. México, D. F.

* Sr. Ing. Pascual Ortíz Rubio... Calle de Ontario 505. Lomas de Chapiltepee, D. F.

Sr. Ing. Narciso Paz........... $3^{n}$ Calle de Hidalgo 30. Pachuea, Hgo.

* Sr. Virgilio Pérez Menchaca..... Prado Sur 240, Jomas de Chapultepee, D. F.

Mr. Adolfo E. Place, M. E..... California Standard Gold Mine Jamentown, Cisl. U. S. A. Corp.

Sr. Ing. Ramiro Robles Ramos.Ciprés 145. México, D. F.

* Sr. Ing. Ramiro Robles, Sr......Mar Blaneo 57. Colonia Anahuae, México, D. F.

Sr. Ing. José Rodríguez Cabo... Av. Insurgentes 590. México, D. F.

* Sr. Ing. Pedro Sánchez Mejorada.Prado Sur 285. México, D. F.

* Sr. Ing Manuel Santillán Vice-! presidente de la Sociedad Geológica Mexicana y Director del Instituto de Geolngía

Av. Insurgentes 577. México, D. F.

* Secretaría de la Economía Nacional.. .. .. ................ Argentina 12. México, .D. F.

Sr. Ing. S. Skewes Samders....Venustiano Carranza 37. México, D. F. 
Nombre.

Domicilio.

Sr. Eduardo Schmitter..................Morelia 101-B. México, D. F:

Sr. Ing. Fugenio Sotomayor.... Calle de Regina 147. México, D. F.

Sr. Ing. Alfredo Terrazas...... Isabel la Católica 40-Desp. 310. México, D. F.

Sr. Ing. Fritz Turban......... Valle de Balderas 126-C, México, D. F.

Sr. Dr. Walter A. Ver Wiebe.... University of Wichita, Wichita, Kansas, U. S. A.

Sr. Ing. Salvador Villamar..... Calle Paloma 10. Tacubaya, D. F.

* Sr. Ing. Juan D. Villarello........... Calle del Pino 129. México, D. F.

* Sr. Ing. Gonzalo Vivar. . . . . . Calle Real de Santiago 2-G. México, D. F.

*: Sr. Dr. Paul Waitz..........Esqs. Trujillo y Partenón, Col. San Alvaro, Popotla, México, D. F.

Sr. Edward Wisser.. ....... Searfe Engineering Cor p.

Sr. Ing. Alfonso Zavada BaldeHeacoek Bldg. Manila, P. I.

nebro. . . . . ....... Calzada de Tacubaya, 95. Tacubaya, D. F. 